\title{
CSEB Student Conference 2016 abstract contest winners
}

\section{Preface}

Health Promotion and Chronic Disease Prevention in Canada was pleased to manage, once again, the student abstract contest for the Canadian Society for Epidemiology and Biostatistics (CSEB) National Student Conference, which was held at the University of Manitoba in June 2016. An editorial panel from the Public Health Agency of Canada judged 17 abstract submissions and selected the top 7 to be published in this issue of the journal.

The editorial panel consisted of the following members:

- Robert Geneau, PhD, Editor-in-Chief

- Heather Orpana, PhD, Associate Scientific Editor

- Michelle Tracy, MA, Managing Editor

The selected abstracts were judged on their originality, clarity, scientific and technical excellence and potential impact.

Since 2009, our journal has collaborated with CSEB to foster publishing opportunities for students. We are proud to collaborate with CSEB again this year and to encourage students in their publishing efforts. On behalf of the journal's editorial team, I would like to thank all students who submitted their abstracts and to congratulate the winners. Having one's abstract published in a peer-reviewed journal is a good place to start in science publishing. We look forward to seeing future submissions of full research articles.

Michelle Tracy, MA

Managing Editor, Health Promotion and Chronic Disease Prevention in Canada 


\section{Music therapy for Alzheimer's patients}

\section{J. Asselstine, BSc (1); V. L. Kristman, PhD (1)}

Introduction: Music therapy (MT) is an attractive, non-pharmacological treatment for many individuals suffering from dementia. It is well established that MT is responsible for many mood-boosting effects in Alzheimer's disease (AD) patients; however it is unclear whether these benefits extend to cognitive outcomes such as enhanced communicative abilities, improved retention and longer attention span. Prior studies focussed on the efficacy of MT for treatment of AD are limited by problematic and inconsistent methodologies, non-specific measurements of outcome and a failure to control for varying levels of dementia and type of MT between study participants. While experts in the field suggest that an active MT model (which involves the participants actively creating music with the music therapist) may be superior to that of a passive MT model (which involves the participants listening to an external source of music), evidence to support this hypothesis is limited.

Objective: The central purpose of this study is to assess MT treatments in a randomized fashion and compare any changes in cognitive functioning that occur between passive, active and no (control) MT groups. The methodology will be modified to make the study feasible at a master's level.

Methods: This study will utilize a three armed randomized control trial (RCT) design. Three long-term care facilities will be selected and randomized to a treatment type: active MT, passive MT, or a control. Participants within each home will be selected based on AD severity determined by the Global Deterioration Scale, as defined by the Alzheimer's Society of Canada. The MT sessions will be conducted by an accredited music therapist twice a week at each location, over a period of six weeks. Three cognitive outcome measures will be collected at each session. Measurements will include the mini-state mental examination (MMSE), the new Music in Dementia Assessment Scale (MiDAS) as proposed by McDermott et al. (2015), and the Alzheimer's disease Assessment Scale (ADAS), with the latter serving as a highly sensitive measurement for cognitive change over time. Following collection, data will be analyzed to explore relationships between treatment type and cognitive outcomes. Each of the three outcomes of interest will be analyzed using multivariate linear regression, with relevant confounding factors included in each model. A $10 \%$ change in the regression coefficient will indicate important confounding effects.

As research at the master's level is constricted by both funding and time, modifications to the proposed methodology are needed. For example, a graduate student rather than an accredited music therapist could conduct the MT sessions.

Results: Results are expected to be collected by August 2017.

Conclusion: The methodological framework utilized in this study will be of interest to other researchers involved with studying MT. Additionally, the results from this study may serve as a reference from which clinicians can make recommendations regarding MT to their patients.

Keywords: music therapy, Alzheimer's, dementia 


\title{
The association between work-related exposures and occupational injuries in the aging United States workforce
}

\author{
N. K. Baidwan, MPH (2); S. Gerberich, PhD (2); H. Kim, ScD (2); A. Ryan, MS (2)
}

Introduction: United States workers aged 55 years and older experience a fatal occupational injury rate that is four times higher than their younger counterparts. Although the rate of nonfatal injuries among older workers is lower than that among younger workers, the subsequent consequences are more severe. Limited research has identified exposures that may be associated with occupational injuries among aging workers.

Objectives: Using a longitudinal study design, we aimed to identify specific work-related exposures associated with occupational injuries in the aging workforce and thus inform injury-prevention strategies.

Methods: Data were obtained from the Health and Retirement Study (HRS), a nationally representative, biennial survey of adults aged 50 years and older and their spouses (regardless of age) in the United States. Of the 20129 people who responded to the HRS in 2004, we selected a study cohort of 7212 respondents who were aged 50 years and above and were working for pay in the year 2004 . This cohort was followed until 2012. We used generalized estimating equations (GEE) with negative binomial-type 2 distribution and Cox regression models to examine relations between work-related exposures (specifically, work-requirements for physical effort, lifting heavy loads, stooping/kneeling/crouching that were measured on a Likert scale) and occupational injuries, while controlling for potential confounders, which were identified based on directed acyclic graphs.

Results: Over the study period, 3.6 occupational injury events per 100 full-time-equivalent workers (FTEs) occurred. The rate of injury events per 100 FTEs was highest among those in the age group 50 to 60 years (4.2) and lowest for those 70 years and older (2.9). Additional univariate models identified high rates of injury among Hispanics, those with low education levels and those employed in occupations like farming/forestry/fishing, construction/extraction and protection services. Multivariable GEE and Cox regression models identified that (controlling for age, gender, race, job type, previous history of occupational injury and job tenure) the risk of experiencing occupational injuries increases significantly with requirements for increasing physical effort, lifting heavy loads, and stooping/kneeling/crouching. The risk of occupational injury nearly tripled for workers whose jobs had these workrequirements all/almost all of the time as compared to those who had them none/almost none of the time.

Conclusion: Priority injury-prevention interventions should target aging workers who are employed in occupations involving these higher work-requirements.

Keywords: injury, occupational health, epidemiology, risk factors

Author reference:

2. Division of Environmental Health Sciences, School of Public Health, University of Minnesota, Minneapolis, MN, US

Correspondence: Navneet Kaur Baidwan; Email: baidw002@umn.edu 


\title{
Chronic pain and self-rated health among middle-aged and older Canadians: an analysis of the Canadian Community Health Survey-Healthy Aging
}

\author{
B. Chireh, MPH (3); C. Waldner, DVM (3,4); C. D’Arcy, PhD (3,5)
}

Introduction: Chronic pain is an important health problem adversely affecting functionality and quality of life. Though self-rated health (SRH) is a major predictor of mortality, its relationship with pain is not well understood. Early detection and treatment of pain can enhance well-being and SRH in seniors.

Objective: In this analysis, we explore 1) how pain and age interact to influence SRH; and 2) provincial variations in SRH across Canada.

Methods: We analyzed cross-sectional data from Statistics Canada's Canadian Community Health Survey-Healthy Aging (n = 30685), which targeted those 45 years and older and was conducted from 2008-12-01 to 2009-11-30. The response rate was 74.4\%. The topics covered included sociodemographics, well-being and chronic diseases. We performed bivariate analyses between each predictor and SRH; unadjusted odds ratios and $95 \%$ confidence intervals are reported. In the second phase of analysis, multivariate model building was used to examine the interaction between predictor variables. We used a two-level logistic regression mixed model consisting of provincial differences (first level) and individual differences (second level). An interclass correlation coefficient was computed to determine the degree of variability regarding seniors' SRH at the provincial level. All statistical analyses were completed in Stata 13 (StataCorp LP, College Station, TX, US) at a $5 \%$ level of significance.

Results: Slightly more than half of respondents $(52.3 \%)$ were female. In the bivariate analysis, those experiencing daily chronic pain were 4.34 times more likely to rate their health as poor $(p<.001)$. In the multivariate analysis, being female was protective $(p<.001)$. Those who reported being depressed, lonely, less educated and/or having a lower income were more likely to rate their health as poor $(p<.001)$. Respondents in Atlantic Canada were more likely to report poorer health. Analgesic users were also 1.56 times more likely to report poorer health $(p<.001)$. The association between pain and SRH was stronger among younger age groups (45-54 years) compared to older age groups ( $\geq 85$ years, with an odds ratio of $4.33[p<.001]$ versus 2.47 [ $p<.001]$ ).

Conclusion: Chronic pain, among other determinants, is associated with SRH. Individuals in rating their health may consider a variety of factors, some of which may not be apparent to health providers. We found the influence of pain on SRH was more pronounced among males and those who reported being depressed, lonely, less educated, and/or having a lower income. Also significant was the impact of pain on SRH among middle-aged Canadians in comparison to other age groups. A traditional east to west gradient in SRH in Canada was also observed in this study.

Keywords: aging, pain, self-rated health, epidemiology

3. School of Public Health, University of Saskatchewan, Saskatoon, Saskatchewan, Canada

4. Western College of Veterinary Medicine, University of Saskatchewan, Saskatoon, Saskatchewan, Canada

5. Department of Psychiatry, University of Saskatchewan, Saskatoon, Saskatchewan, Canada 


\title{
The effect of the Roots of Empathy program on the use of psychotropic medications among youth in Manitoba
}

\author{
L. Dahl, MSc (6); M. Chartier, PhD (6,7); R. Fransoo, PhD (6,7); B. Tefft, PhD (8)
}

Introduction: The Roots of Empathy (ROE) program was piloted in Manitoba schools during 2001 and has been implemented every year since to interested public, band-operated and private school divisions and communities. The aim of the program is to strengthen essential social skills through empathy development in children from kindergarten to Grade 8. Research has demonstrated the program's ability to effectively achieve these objectives; however, more distal and unintended outcomes of the program have not been studied. The program may improve mental health and has the potential, therefore, to decrease the use of psychotropic medications in youth.

Objectives: The objective of this study is to determine if the Manitoba Roots of Empathy program is reducing the risk of youth being dispensed psychotropic medication if they participated in the program as children.

Methods: This study uses administrative health, education and social service records for youth in Manitoba to estimate the effect of the ROE program on future psychotropic medication use. Children who participated in ROE between the 2002/03 and 2012/13 school years were matched to children who did not receive ROE through a combination of hard matching on key variables and propensity score matching approaches. Only children who were not dispensed a psychotropic medication in the year prior to the start of followup were eligible for study. Incomplete 10:1 matching without replacement resulted in 16815 children in the control group and 5563 children in the ROE group. Youth from both groups will be followed from the conclusion of their ROE program for the first occurrence of being dispensed a medication from five subclasses of psychotropic medications: (1) antidepressants, (2) anxiolytics, (3) psychostimulants, (4) antipsychotics, and (5) hypnotics and sedatives. Kaplan-Meier survivor curves and Cox proportional regression models will be used to compare and describe the survival experience between the two groups. Analyses will be conducted on a composite outcome for being dispensed any psychotropic medication, as well as separate analyses for each of the subclasses of psychotropic medications.

Results: The study is expected to be completed by December 2016.

Conclusion: This study may provide novel insights regarding an unanticipated outcome of the ROE program that extends beyond the proximal outcomes that have established the program's success. Measuring the future use of psychotropic medications in youth may demonstrate a lasting effect of the program and its effectiveness to promote mental health among youth.

Keywords: program effectiveness evaluation, psychotropic medication, youth

Author references:

6. Department of Community Health Sciences, Max Rady College of Medicine, Rady Faculty of Health Sciences, University of Manitoba, Winnipeg, Manitoba, Canada

7. Manitoba Centre for Health Policy, Rady Faculty of Health Sciences, University of Manitoba, Winnipeg, Manitoba, Canada

8. Department of Psychology, Faculty of Arts, University of Manitoba, Winnipeg, Manitoba, Canada

Correspondence: Lindsey Dahl; Email: umdahllt@myumanitoba.ca 


\title{
Specialized care and recurrent traumatic brain injury: a retrospective cohort study
}

\author{
O. Lasry, MDCM (9,10); J. Marcoux, MD (10); D. Buckeridge, MD (9)
}

Introduction: Many traumatic brain injury (TBI) patients suffer a recurrent TBI (rTBI). These patients are at a higher risk of poor cognitive and functional outcomes compared to patients with a single injury. However, interventions that may mitigate the risk of rTBI are currently unknown.

Objective: This study aimed to determine whether the care provided for an index mild TBI (mTBI) in neurotrauma centre emergency departments (specialized care) is associated with a lower risk of rTBI at one-year follow-up when compared to the care provided in non-neurotrauma centre emergency departments (non-specialized care).

Methods: A retrospective cohort study of all patients incurring mTBI and being treated in an emergency department from 1998 to 2014 was completed. Each patient had a follow-up period of up to one year. Administrative data for a $25 \%$ random sample of the Greater Montréal area were used to ascertain cases, outcomes and covariates. A time-to-event analysis was conducted using a Cox proportional hazards model, taking into account the time-dependent effects of the type of care received. Important confounders were included in the model to adjust for confounding. A sensitivity analysis using an instrumental variable (differential distance between a patient's closest neurotrauma and non-neurotrauma centre) was used to assess for residual confounding.

Results: During the study period there were 24292 (19 516 non-specialized care and 4776 specialized care) mTBI patients that presented to an emergency department. A total of 1384 rTBI (5.7\%) cases occurred during a one-year follow-up of each patient. After adjusting for confounders and the time-dependent effect of the type of care received, patients treated in neurotrauma centre emergency departments had a $36 \%$ reduction in the hazard of rTBI with a hazard ratio (HR) of 0.64 (95\% CI: 0.51-0.80) in the first week after the index injury compared to patients treated in non-specialized emergency departments. This protective effect persisted for the first six weeks after the index injury. Over the one-year follow-up period, the overall reduction in the hazard of rTBI was $21 \%$ $(\mathrm{HR}=0.79,95 \% \mathrm{CI}: 0.68-0.91)$ for patients treated in the specialized care setting. The instrumental variable sensitivity analysis supported these findings.

Conclusion: The treatment of mTBI patients in the emergency department of neurotrauma centres is associated with an important decrease in the occurrence of rTBI. Further research that identifies the mediators of this association is warranted so that specific interventions that lower the risk of rTBI can be implemented.

Keywords: traumatic brain injury, recurrence, specialized care

Author references:

9. Department of Epidemiology, Biostatistics and Occupational Health, McGill University, Montréal, Quebec, Canada

10. Department of Neurology and Neurosurgery, McGill University Health Centre, McGill University, Montréal, Quebec, Canada

Correspondence: Oliver Lasry; Email: oliver.lasry@mail.mcgill.ca 


\title{
Influence of a pay-for-performance program on the care of diabetic patients by family physicians in New Brunswick
}

\author{
E. LeBlanc, BSc (11,12); M. Bélanger, PhD (11,12,13); V. Thibault, BSc (11,12); L. Babin, MD (11,12,13); \\ B. Greene, MN (14); S. Halpine, PhD (14); M. Mancuso, MBA (15)
}

Introduction: With increasing costs associated with diabetes management, many jurisdictions are using pay-for-performance programs to entice family physicians to follow practice guidelines when caring for their diabetic patients. The impact of such programs on the health of patients is unknown.

Objective: The objective of this study was to determine whether hemoglobin A1C (A1C) of diabetic patients was improved following the introduction of an incentive program in New Brunswick in 2010. Two outcomes were considered: the probability of having had at least two A1C tests per year and the mean A1C of patients.

Methods: Administrative data from all diabetic patients $(n=83580)$ with A1C tests were acquired from the New Brunswick Department of Health and patients' respective fee-for-service family physicians $(\mathrm{n}=583)$. Both repeat-cross-sectional and cohortbased (2005-2014) analyses were conducted. Patients were divided in two groups depending on whether their family physician had claimed the incentive or not. Multivariate analyses were conducted to compare pre- and post-incentive period for probability of having had two tests per year (logistic) and mean A1C (linear). The same outcomes were compared between patients for whom an incentive was claimed and those for whom it was not claimed after 2010.

Results: Family physicians who were already prescribing at least two A1C tests per year before the implementation of the incentive program had $51 \%$ greater odds than other physicians to continue to do so after the implementation (99\% CI: 1.44-1.57). Patients followed by a family physician claiming the incentive had 33\% greater odds of receiving at least two A1C tests per year (99\% CI: 1.24-1.43). However, there were no differences in annual A1C means between sub-groups studied.

Conclusion: This analysis suggests that implementation of an incentive program led physicians to provide better follow-up care to patients with diabetes. However, the incentive program has not been associated with differences in glycemic control. These results suggest that although incentive programs may help physicians provide better care to their patients with diabetes, these programs may not be sufficient to result in optimal glycemic control. Further analyses are needed to confirm these results, to estimate the combined effects of incentive programs with other interventions and to assess the cost-benefit of such interventions.

Keywords: diabetes, incentive, family physician

\section{Author references:}

11. Faculty of Medicine and Health Sciences, Université de Sherbrooke, Sherbrooke, Quebec, Canada

12. Centre de formation médicale du Nouveau-Brunswick, Université de Moncton, Moncton, New Brunswick, Canada

13. Vitalité Health Network, Moncton, New Brunswick, Canada

14. New Brunswick Department of Health, Fredericton, New Brunswick, Canada

15. New Brunswick Health Council, Moncton, New Brunswick, Canada

Correspondence: Emilie LeBlanc; Email: Emilie.R.Leblanc@USherbrooke.ca 


\title{
Children with autism spectrum disorder in Manitoba: prevalence, population characteristics and psychotropic medication use
}

\author{
L. Vehling, MSc (6); M. Brownell, PhD (6,7); J. Kaufert, PhD (6); N. C. Nickel, PhD (6,7); S. Alessi-Severini, PhD (16)
}

Introduction: Autism spectrum disorder (ASD) is a neurodevelopmental disability diagnosed in an increasing number of children. Psychotropic medication use has also increased among Canadian children and may be more common among children with intellectual disabilities. However, psychotropic medications are not strongly recommended for use among children and have been associated with significant adverse events. ASD has few effective treatment options, yet psychotropic medications are used to manage challenging behaviours in some children with ASD.

Objectives: This study describes ASD prevalence and use of psychotropic medications in the pediatric population of Manitoba. Populations of children with and without ASD are described and compared to determine difference in psychotropic medication use and population characteristics.

Methods: Administrative data from the Repository at the Manitoba Centre for Health Policy were used to create a cohort of children born in Manitoba. Diagnoses of ASD were based on medical claim records, hospital abstracts or special education funding data.

Results: Between 2010 and 2014, 3079 Manitoba children aged 0 to 14 years had an ASD diagnosis (1\% prevalence). Among children with ASD aged 0 to 18 years, $80 \%$ are boys, almost $60 \%$ were diagnosed before age 5 , with $85 \%$ diagnosed before age 10 , and almost $50 \%$ received special education funding. Close to $50 \%$ of all children with ASD received a psychotropic prescription before age 18 , compared to only $12 \%$ in the population of children in Manitoba without ASD or an intellectual disability. Children with ASD are more likely than those in the general population of children to live in Winnipeg, be involved with child welfare services and receive psychotropic medications.

Conclusion: In Manitoba, ASD is diagnosed in preschool or early school years, when treatment and services can have the greatest impact on functioning. Children with ASD are more likely to use psychotropic medications and have different population characteristics when compared to children in the general population. This epidemiological information is important to understand risk factors, assess current treatment strategies and direct future intervention planning.

Keywords: autism, psychotropic medications, population health

Author references:

6. Department of Community Health Sciences, Max Rady College of Medicine, Rady Faculty of Health Sciences, University of Manitoba, Winnipeg, Manitoba, Canada

7. Manitoba Centre for Health Policy, Rady Faculty of Health Sciences, University of Manitoba, Winnipeg, Manitoba, Canada

16. College of Pharmacy, Rady Faculty of Health Sciences, University of Manitoba, Winnipeg, Manitoba, Canada 